\title{
Circularly Polarized States Spawning from Bound States in the Continuum
}

\author{
Wenzhe Liu, ${ }^{1,2}$ Bo Wang, ${ }^{1,2}$ Yiwen Zhang, ${ }^{1,2}$ Jiajun Wang, ${ }^{1,2}$ Maoxiong Zhao, ${ }^{1,2}$ \\ Fang Guan, ${ }^{1,2}$ Xiaohan Liu, ${ }^{1,2}$ Lei Shi, ${ }^{1,2, *}$ and Jian $\mathrm{Zi}^{1,2, \dagger}$ \\ ${ }^{1}$ State Key Laboratory of Surface Physics, Key Laboratory of Micro- and Nano-Photonic Structures (Ministry of Education) and \\ Department of Physics, Fudan University, Shanghai 200433, China \\ ${ }^{2}$ Collaborative Innovation Center of Advanced Microstructures, Nanjing University, Nanjing 210093, China
}

(Received 31 March 2019; published 12 September 2019)

\begin{abstract}
Bound states in the continuum in periodic photonic systems like photonic crystal slabs are proved to be accompanied by vortex polarization singularities on the photonic bands in the momentum space. The winding structures of polarization states not only widen the field of topological physics but also show great potential that such systems could be applied in polarization manipulating. In this Letter, we report the phenomenon that by in-plane inversion $\left(C_{2}\right)$ symmetry breaking, pairs of circularly polarized states could spawn from the eliminated bound states in the continuum. Along with the appearance of the circularly polarized states as the two poles of the Poincaré sphere together with linearly polarized states covering the equator, full coverage on the Poincaré sphere could be realized. As an application, ellipticity modulation of linear polarization is demonstrated in the visible frequency range. This phenomenon provides a new degree of freedom in modulating polarization. The $C$ points could also find applications in light-matter interactions. Further studying and manipulating the reported polarization singularities may lead to novel phenomena and physics in radiation modulating and topological photonics.
\end{abstract}

DOI: 10.1103/PhysRevLett.123.116104

Polarization is one of electromagnetic wave's most essential properties. Controlling the polarization is found very important in a lot of fields, such as 3D imaging [1], optical communication [2], and quantum optics [3]. Recently, great attention has been paid to modulate polarization of light with compact structures such as metasurfaces [4-7] instead of classical wave plates and polarizers, which are more applicable in on-chip devices. On the other hand, the application of another kind of structure, photonic crystal (PHC) slab, in modulating polarization, is capturing interest [8-11]. Their simplicity in fabrication, designable band structures, and complex polarization features in the momentum space which are topologically linked with bound states in the continuum [10-27] are favorable for polarization modulation. However, the reported PHC slabs only support nearly linearly polarized resonances which only cover a small area on the Poincaré sphere. The coverage on the Poincaré characterized the polarization properties of the system. Small coverage without the two poles (circular states of polarization) indicates its limited capability in full Stokes polarization modulating. In this Letter, we report that, by

Published by the American Physical Society under the terms of the Creative Commons Attribution 4.0 International license. Further distribution of this work must maintain attribution to the author(s) and the published article's title, journal citation, and DOI. breaking the in-plane inversion $\left(C_{2}\right)$ symmetry of a PHC slab, the at- $\Gamma$ BICs also known as vortex polarization singularities ( $V$ points) $[12,13,15-17,21,24,28]$ on photonic bands will be eliminated [22]. As the singularities are broken, the winding of the main axis of the polarization states are preserved, leading to generation of pairs of circularly polarized states ( $C$ points) near the $\Gamma$ point. With a line of linearly polarized states ( $L$ line) enclosing the position of the original BIC, the generation of $C$ points would even enable full coverage on the Poincaré sphere. Its application in ellipticity modulation of light is demonstrated.

For a 2D PHC slab, there would be a series of Bloch resonant modes with different frequencies $f$ and wave vectors $k$ forming photonic bands. Radiation polarization states of these modes on an arbitrary band could be projected into the structure plane and mapped onto the Brillouin zone (BZ), which defines a polarization field in the momentum space $[12,13,15-17,21,24,28]$. These Bloch modes are mostly radiative, unless destructive interference or symmetry mismatch makes them nonradiative, i.e., BICs. The BICs appear as vortex singularities ( $V$ points) in this field. To characterize the polarization properties of the system, we can map the polarization states of the Bloch modes onto the Poincaré sphere of which the coordinates are specified by Stokes parameters $S_{0}, S_{1}, S_{2}$, and $S_{3}$. In this language, those nonradiative singularities will have zero values of Stokes parameters. Thus, they are also singularities in the normalized Stokes parametric space, shown as the left panel of Fig. 1. Proven in Ref. [9], the polarization 


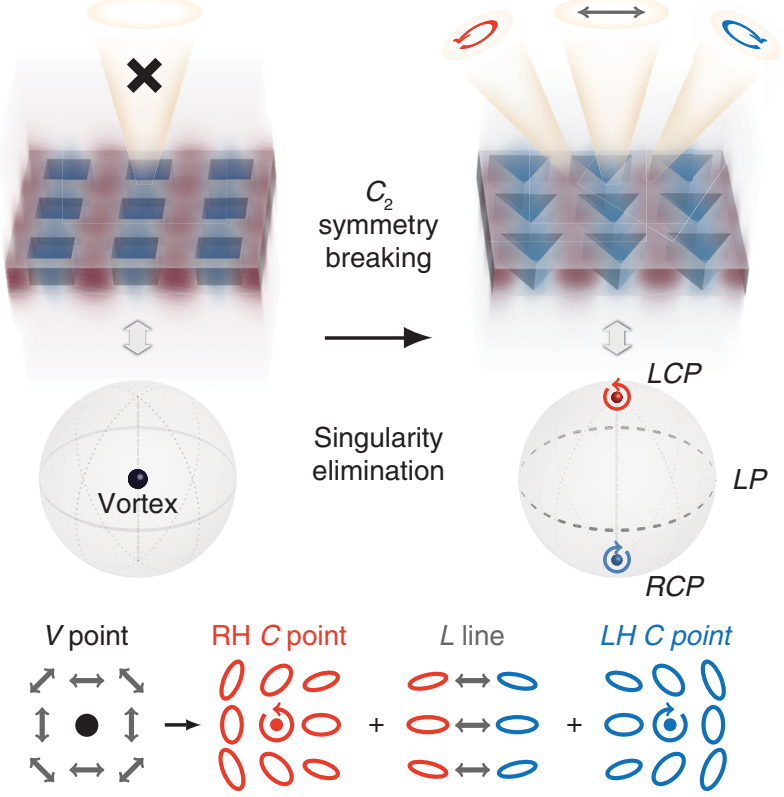

FIG. 1. Schematic view of how circularly polarized states ( $C$ points) spawn from a bound state in the continuum when in-plane inversion $\left(C_{2}\right)$ symmetry is broken. $C$ points and lines of linearly polarized states ( $L$ lines) could be found near $\Gamma$ point. The asymmetric field of the mode induces extra in-plane multipole moments. $L(R) C P$ : left(right)-handed circular polarization; $\mathrm{L}(\mathrm{R}) \mathrm{H}$ : left(right)-handed.

states of radiative modes are almost linear in the whole BZ of a 2D PHC slab with $C_{2}$ symmetry, of which the low ellipticity results from the perturbative non-Hermiticity. As a consequence, the corresponding polarization map projected from the BZ onto the normalized Poincare sphere should be a belt near the equator plus a singularity. With a large area including two poles not covered, it will limit the applications of 2D PHC slabs such as polarization modulating. Recently, it was known that the symmetry-protected BICs at the $\Gamma$ point origin from the multipole moment perpendicular to the structure plane in a $C_{2}$ symmetric system $[17,22,24,27]$. If the $C_{2}$ symmetry is broken, the electromagnetic field will be perturbed as shown in Fig. 1, and nonzero multipole moments lying in the structure plane would be induced. As researched in singular optics [29-36], $V$ points $\left(S_{0}, S_{1}, S_{2}, S_{3}=0\right)$ result from the collision of $C$ points $\left(S_{1}, S_{2}=0\right)$ and $L$ lines $\left(S_{3}=0\right)$ $[33,37,38]$. Thus with the multipolar perturbation induced, the at- $\Gamma$ integer-charged $V$ points will decompose into pairs of half-charged $C$ points and $L$ lines (illustrated in the right panel of Fig. 1). The aforementioned limitation on ellipticity will be broken.

To verify the physical picture above, we designed freestanding 2D PHC slabs and studied the phenomenon in theory and experiment. The slabs here are made of silicon nitride $\left(\mathrm{Si}_{3} \mathrm{~N}_{4}\right.$, refractive index $\left.\sim 2.02\right)$. The thicknesses $t$ are chosen to be $100 \mathrm{~nm}$. Square lattices of holes with a period $a=450 \mathrm{~nm}$ are etched on the slabs with the original shape square. And there is no substrate, which maintains the up-down mirror symmetry and guarantees a large refractive index difference making the phenomenon apparent. Absence of the substrate also avoids extra diffractive channels which will affect the phenomenon [39]. Then, to break the $C_{2}$ symmetry, the squares are transformed to isosceles trapezoids with their area $S=$ $S_{0}=247 \mathrm{~nm} \times 247 \mathrm{~nm}$ unchanged. The left-right mirror line is maintained to exclude the effect of breaking mirror symmetry, which simplifies the physics. During the transformation, the height $h$ and the baseline length $L$ of any trapezoid are kept equal $(L=h)$. The asymmetry parameter $\alpha$ here is then defined as the ratio of the reduced topline length $2 \Delta L$ to the baseline length $L$ [see Fig. 2(d)]. Increasing $\alpha$ from 0 to 1 would transform the holes from squares to isosceles triangles, while $L=\sqrt{2 S_{0} /(2-\alpha)}$.

We first focus on the at- $\Gamma$ BIC of band $\mathrm{TE}_{2}$ for example. Applying finite element method calculation, we firstly confirmed that the quality factors of the at- $\Gamma$ states on band $\mathrm{TE}_{2}$ obey the law $\alpha^{-2}$ when the perturbation is small, which is proved in Ref. [22]. Results are included in Supplemental Material [40]. Subsequently, we obtained the projected radiation polarization states $\left(d_{x}, d_{y}\right)$ on band $\mathrm{TE}_{2}$ to see the generation of $C$ points. The band structures and polarization maps near the $\Gamma$ point of systems with different asymmetry parameters $\alpha=0,0.1$, and 1 are plotted in Figs. 2(a)-2(c). We can see in Fig. 2(a) that when $\alpha=0$, the polarization states are close to linear, and there is a $V$ point (BIC) at the $\Gamma$ point (marked as a black dot). When $\alpha$ slightly increases to 0.1 , shown in Fig. 2(b), the vortex is broken and a pair of $C$ points with opposite chirality (the two poles) appear very close to the $\Gamma$ point [marked as red $(L C P)$ and blue $(R C P)$ dot], clamping an $L$ line. This indicates that the areas around the two poles of the Poincare sphere are covered. The $C$ points spawn in the $\Gamma-X$ direction due to the maintained mirror symmetry. It should be noted that we have an $L$ line far away from the $\Gamma$ point enclosing the original at $\Gamma V$ point in our system, as shown in the Supplemental Material [40]. This $L$ line corresponds to the entire equator of the Poincare sphere, and, due to continuity, the full Poincaré sphere is thus covered. To prove this, we projected circular loops in the $\mathrm{BZ}$ around the $\Gamma$ point onto the normalized Poincare sphere under the condition $\alpha=0.1$. We find that the larger area the loop in the BZ encloses [shown in Fig. 2(e), left panel], the closer the loop on the Poincare sphere is to the equator [shown in Fig. 2(e), right panel]. We can see the coverage approaching the full Poincare sphere. Note that there is a point on the equator pinned on every loop corresponding to the clamped $L$ line protected by the left-right mirror symmetry. As the perturbation is larger $[\alpha=1$, shown in Fig. 2(c)], the $C$ points will move away from the $\Gamma$ point. A larger distance between $C$ points will simplify the experimental observation. It is worth mentioning that breaking other types of 


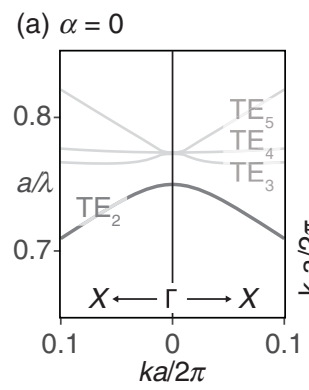

(d)

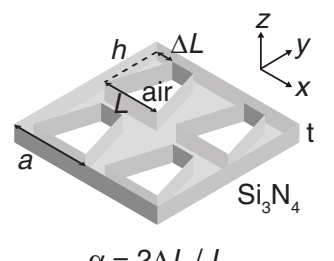

(b) $\alpha=0.1$

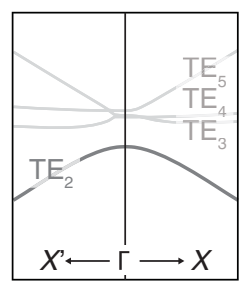

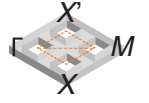

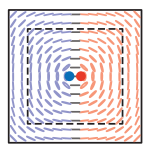

(c) $\alpha=1$

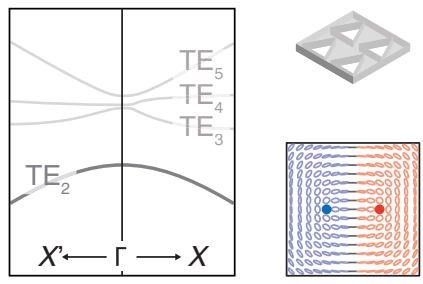

(e)
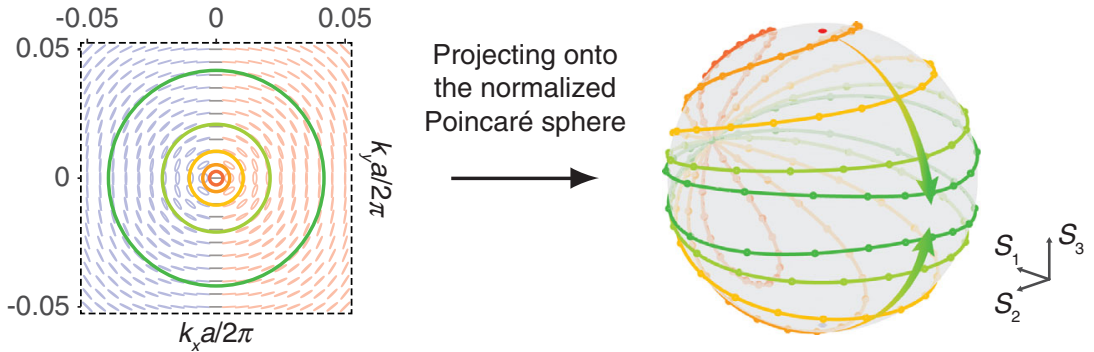

FIG. 2. (a)-(c) The TE-like band structures and polarization maps in the vicinity of the $\Gamma$ point of structures with different values of $\alpha$. We focus on band $\mathrm{TE}_{2}$, which is marked with bold dark line on the band structure diagram. On the polarization maps, the polarization states are represented by ellipses of which the red (blue) color corresponds to left-handed (right-handed) states. The enlarged map of the enclosed area in (b) will be shown in (e). (d) A schematic view of the structure and the definition of the asymmetry parameter $\alpha$. (e) The circular loops of polarization states mapped from the Brillouin zone (BZ) to the Poincaré sphere. The radius of the loops are 0.0025, $0.005,0.01,0.02$, and 0.04 from orange to green. The data points are sampled per 10 degrees on every loop in the BZ.

symmetry, e.g., the up-down symmetry, could only break off- $\Gamma$ BICs while the in-plane $C_{2}$ symmetry breaking would eliminate both at- $\Gamma$ and off- $\Gamma$ BICs due to different kinds of perturbation they induce. Detailed discussion on this is included in Supplemental Material [40]. The mechanism to manipulate the polarization singularities in the momentum space deserves further study, and may find fascinating applications.

When taken one step further, the reported phenomenon is promised to be found on any band. We analyzed the bands $\mathrm{TE}_{2}, \mathrm{TM}_{2}$, and $\mathrm{TM}_{5}$ of the above-studied 2D PHC slab with $\alpha=1$, finding the phenomenon not only common but also giving different topological polarization configurations of $C$ points [29-34]. Three typical configurations were found: a "lemon" is found on $\mathrm{TE}_{2}$, a "star" is found on $\mathrm{TM}_{2}$, and a "monstar" is found on $\mathrm{TM}_{5}$. The band structures along $\Gamma-X$ and $\Gamma-X^{\prime}$ are plotted in Fig. 3(a). We mapped the polarization states on the studied bands by simulation, and connected the main axis of the polarization ellipses to present the polarization field in form of streamlines, aiming to show the different configurations clearly in Fig. 3(b). The $C$ points are marked as red and blue dots. Different configurations on different bands give us much more freedom in designing the eigenmodes with the desired polarization states at desired incident or radiating directions.

For an experimental observation of this phenomenon in the visible frequency range, we fabricated the simulated PHC slabs. The designed hole array is etched on a freestanding $\mathrm{Si}_{3} \mathrm{~N}_{4}$ layer applying reactive-ion etching with the assistance of the mask poly(methyl methacrylate) layer etched by the electron beam lithography. For optical measurements, we applied our home-made momentumspace imaging spectroscopy system [16] to obtain their polarization-dependent angle-resolved transmittance spectra. Under $L C P$ (and $R C P$ ) incidence, the spectra [Fig. 4(c)] of the $\alpha=0$ sample shows no diminishing point other than nonexcited BICs at the $\Gamma$ point. In contrast, the at $-\Gamma$ states of

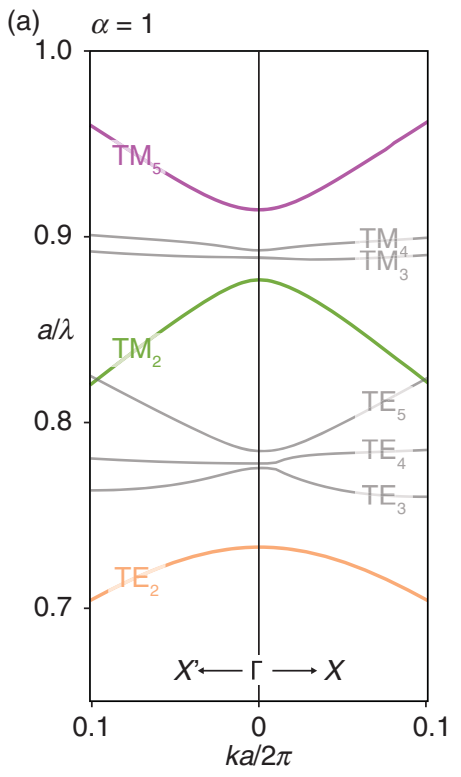

(b) $\quad \begin{array}{lll}-0.07 & 0 & 0.07\end{array}$

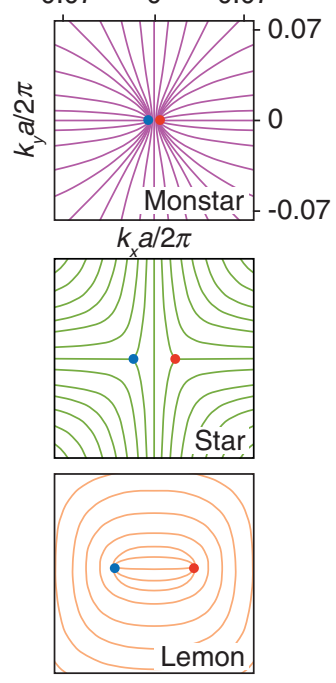

FIG. 3. (a) The band structures along $\Gamma-X$ and $\Gamma-X^{\prime}$ near the $\Gamma$ point. (b) Different configurations of polarization main axis near the $\Gamma$ point on bands $\mathrm{TE}_{2}$ (bottom, "lemon"), $\mathrm{TM}_{2}$ (middle, "star"), $\mathrm{TM}_{5}$ (top, "monstar") of the structure with $\alpha=1$. The different colors correspond to the colored bands in (a). 


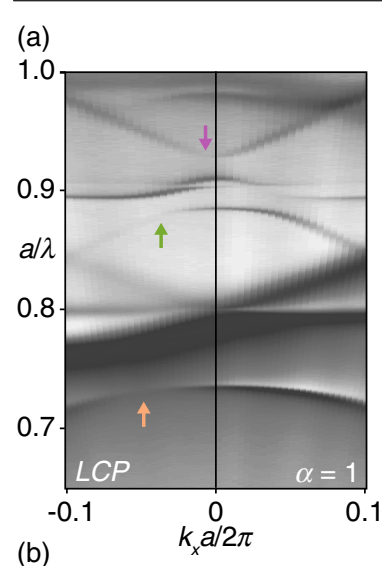

(c)
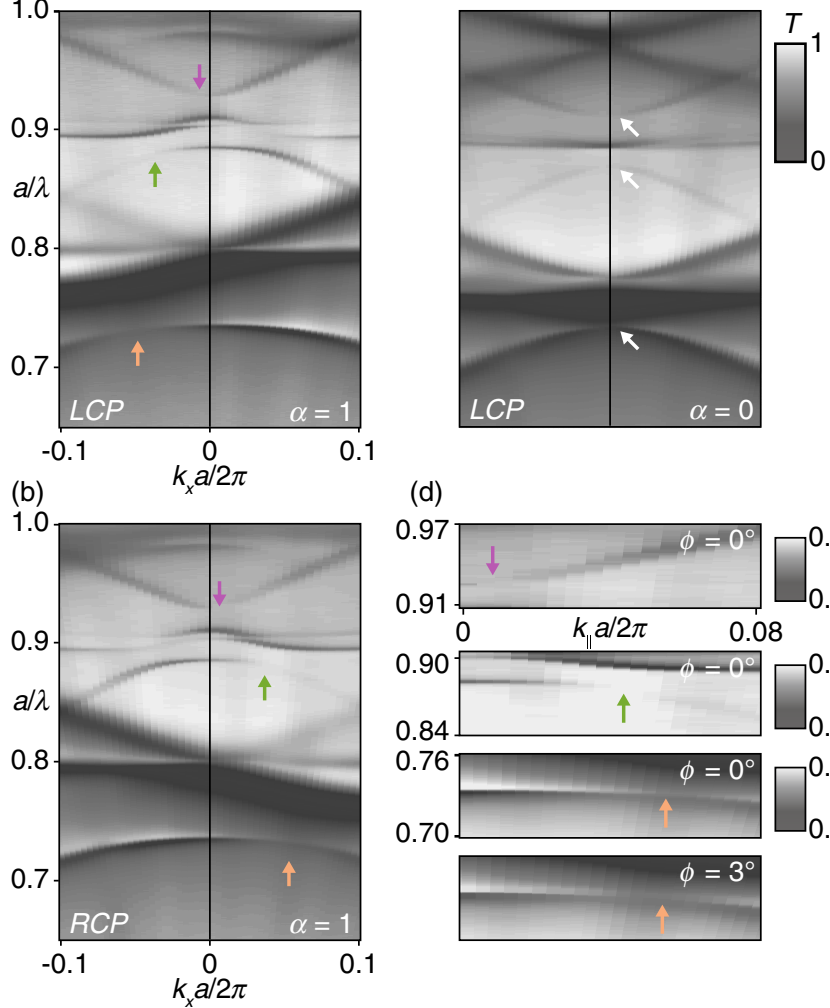

(d)
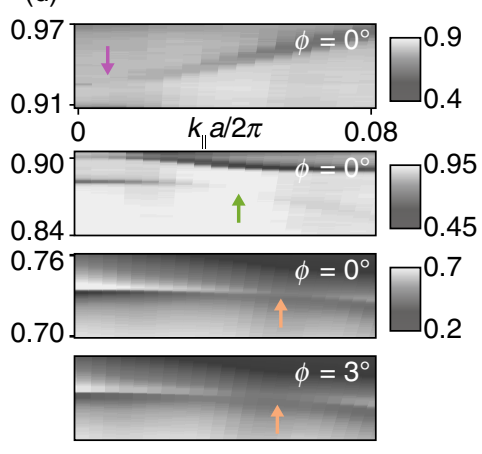

FIG. 4. The measured angle-resolved transmittance spectra in the visible range under circularly polarized incidence. (a) The spectra of the $\alpha=1$ sample, LCP incidence. Vanished regions are pointed out by arrows, implying the existence of $C$ points after breaking the $C_{2}$ symmetry. The colors correspond to the colored bands in Fig. 3. (b) The spectra of the $\alpha=1$ sample, $R C P$ incidence. (c) The spectra of the $\alpha=0$ sample, LCP incidence. The vanished regions marked by white arrows are the BICs. (d) Enlarged plots of the vanished regions in (c). Note that the $C$ points on band $\mathrm{TE}_{2}$ are in the direction $\phi \sim \pm 3^{\circ}$ to $\Gamma-X$; thus the spectra along this direction are plotted.

the $\alpha=1$ sample [Fig. 4(a)] are radiative and some regions near the $\Gamma$ point on the bands are diminished instead under $L C P$ incidence. The nonexcited states in these regions are hence $\mathrm{RH}$ polarized with $C$ points at the center. Changing the incidence to $R C P$, the diminished regions switch to the other side of the BZ, meaning another $C$ point with opposite chirality [Fig. 4(b)]. The enlarged plots of the diminished regions are shown in Fig. 4(d), with detailed $R C P$ spectra showing the $C$ point included in the Supplemental Material [40]. The existence of the $C$ points are proven and agree with the simulations.

Basing on the angle-resolved polarization dependent spectra we measured, we mapped transmittance along band $\mathrm{TE}_{2}$ as a function of $f$ and $k$ under $L C P$ and $R C P$ incidence to observe the $C$ points and the coverage on Poincare sphere more clearly, plotted in Fig. 5(a). Note that, the maps not only cover the BZ, but also span spectral ranges. Changing the period to $400 \mathrm{~nm}$, the bottom line length of
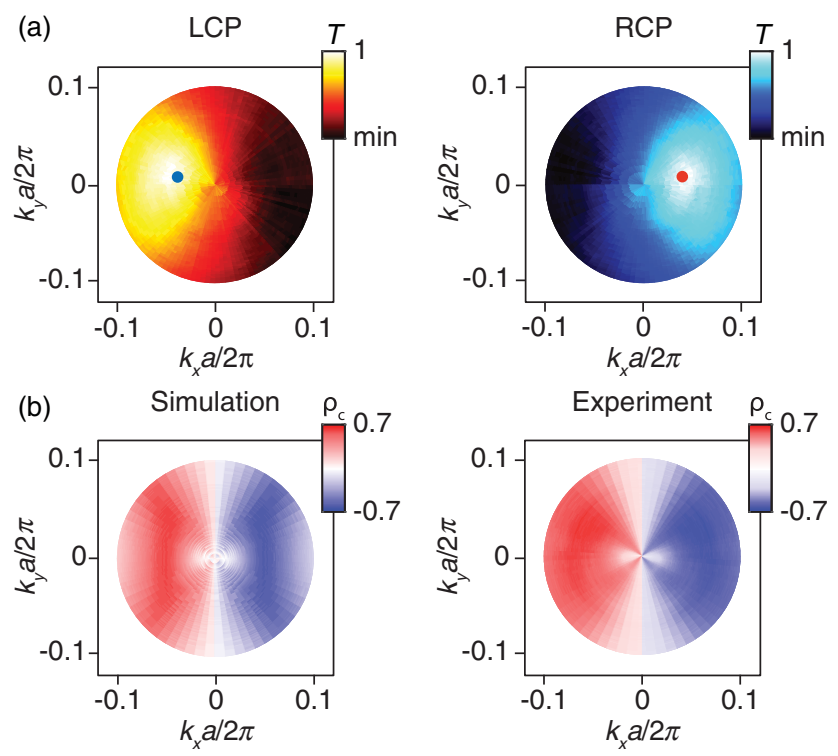

FIG. 5. (a) Experimental observation of the coverage on Poincaré sphere presented in the form of transmittance maps along the band $\mathrm{TE}_{2}$ in the visible frequency range. (b) Simulated and measured ellipticity maps of transmitted polarization states under $p$ incidence on the same band as in (a).

the triangle to $300 \mathrm{~nm}$, the thickness of the slab to $215 \mathrm{~nm}$, we were able to suppress the effect of Fano resonances, and the reflectance (1-transmittance) in such a case approximately corresponds to the inner product of the projected polarization state and the incident circular polarization (detailed in the Supplemental Material [40]). On either map, we can see one spot where the transmittance approaches 1 , indicating a $C$ point not coupling with the incident light. From the map, the distribution of polarization states is exhibited, and the coverage approaching full Poincare sphere is directly observed in a rather small range of the BZ. We also extracted the polarization states from the spectra, which is detailed in the Supplemental Material [40].

The coverage approaching full Poincaré sphere gives wider choices in modulating polarization with PHC slabs. Here in the visible range, we demonstrate the application in modulating the ellipticity $\rho_{c}=S_{3} / S_{0}$. $\left(S_{0}, S_{3}\right.$ : the first and fourth Stokes parameters.) In Fig. 5(b), we plotted the ellipticity map of the completely polarized transmitted light on resonances of the band $\mathrm{TE}_{2}$, applying the sample measured in Fig. 5(a). $\rho_{c}$ here is calculated from the onresonance transmittance measured with $p$-, $s$ - and $\pm 45^{\circ}$ polarized analyzers. As we can see from Fig. 5(b), the $p$ polarized incident light $\left(\rho_{c}=0\right)$ is filtered to be elliptically polarized, of which $\rho_{c}$ reaches 0.7 at most. The results of experiments (right panel) match up with rigorous coupled wave algorithm simulations (left panel). Choosing the right parameters and with higher fabrication precision, the linearly polarized light could be perfectly filtered to be circularly polarized light in theory (detailed in the Supplemental Material [40]). To be noted that, this effect 
of splitting the two circular polarizations can be viewed as a result of strong orbit-spin coupling induced by $C_{2}$ symmetry breaking, and similar phenomena in monochromatic beams also induced by symmetry breaking have been shown previously $[41,42]$.

In conclusion, we reported the phenomenon that by breaking the in-plane inversion symmetry $\left(C_{2}\right)$ of a $2 \mathrm{D}$ PHC slab, the nonradiative vortex singularities at the $\Gamma$ point will be eliminated. As a consequence, $C$ points with different polarization configuration could be found near the $\Gamma$ point. Together with an $L$ line looping around, the full Poincaré sphere coverage can be realized. We theoretically verified and experimentally observed the generation of $C$ points and the coverage approaching full Poincaré sphere. This phenomenon enriches the knowledge on polarization properties of 2D PHC slabs in the momentum space, and could be linked with topological photonics. The abundant dynamics of various polarization singularities in the momentum space may lead to novel physical phenomena both in radiation and in-plane transportation, which can be combined with valleytronic materials in applications. The coverage on the Poincare sphere offers us new latitude to modulate polarization with 2D PHC slabs, which may be applied in polarization filtering, vector beam generating or quantum optics.

We thank Dr. Ang Chen, Professor Chao Peng, Professor Chia Wei Hsu, Professor Dezhuan Han, Professor Ling Lu, Professor Meng Xiao, Dr. Haiwei Yin, and Professor Shaoyu Yin for helpful discussions. The work was supported by 973 Program and China National Key Basic Research Program (Grants No. 2015CB659400, No. 2016YFA0301100, No. 2016YFA0302000, and No. 2018YFA0306201) and National Science Foundation of China (Grants No. 11774063, No. 11727811, No. 91750102, and No. 11604355). The research of L.S. was further supported by the Science and Technology Commission of Shanghai Municipality (Grants No. 17ZR1442300 and No. 17142200100).

W. L., B. W., and Y.Z. contributed equally to this work.

"1shi@fudan.edu.cn

jzi@fudan.edu.cn

[1] M. Pircher, E. Goetzinger, R. Leitgeb, and C. K. Hitzenberger, Phys. Med. Biol. 49, 1257 (2004).

[2] G. Keiser, Optical Fiber Communications (Wiley, New York, 2003).

[3] L. Mandel and E. Wolf, Optical Coherence and Quantum Optics (Cambridge University Press, Cambridge, England, 1995).

[4] Y. Zhao and A. Alù, Phys. Rev. B 84, 205428 (2011).

[5] S. B. Glybovski, S. A. Tretyakov, P. A. Belov, Y. S. Kivshar, and C. R. Simovski, Phys. Rep. 634, 1 (2016).
[6] S. Kruk, B. Hopkins, I. I. Kravchenko, A. Miroshnichenko, D. N. Neshev, and Y. S. Kivshar, APL Photonics 1, 030801 (2016).

[7] S. Kruk and Y. Kivshar, ACS Photonics 4, 2638 (2017).

[8] S. V. Lobanov, T. Weiss, N. A. Gippius, S. G. Tikhodeev, V. D. Kulakovskii, K. Konishi, and M. Kuwata-Gonokami, Opt. Lett. 40, 1528 (2015).

[9] C. W. Hsu, B. Zhen, M. Soljačić, and A. D. Stone, arXiv:1708.02197.

[10] Y. Guo, M. Xiao, and S. Fan, Phys. Rev. Lett. 119, 167401 (2017).

[11] Y. Guo, M. Xiao, Y. Zhou, and S. Fan, Adv. Opt. Mater. 7, 1801453 (2019).

[12] C. W. Hsu, B. Zhen, J. Lee, S.-L. Chua, S. G. Johnson, J. D. Joannopoulos, and M. Soljačić, Nature (London) 499, 188 (2013)

[13] B. Zhen, C. W. Hsu, L. Lu, A. D. Stone, and M. Soljačić, Phys. Rev. Lett. 113, 257401 (2014).

[14] Y. Yang, C. Peng, Y. Liang, Z. Li, and S. Noda, Phys. Rev. Lett. 113, 037401 (2014).

[15] E. N. Bulgakov and D. N. Maksimov, Phys. Rev. A 96, 063833 (2017).

[16] Y. Zhang, A. Chen, W. Liu, C. W. Hsu, B. Wang, F. Guan, X. Liu, L. Shi, L. Lu, and J. Zi, Phys. Rev. Lett. 120, 186103 (2018).

[17] H. M. Doeleman, F. Monticone, W. den Hollander, A. Alù, and A. F. Koenderink, Nat. Photonics 12, 397 (2018).

[18] Y. Song, N. Jiang, L. Liu, X. Hu, and J. Zi, Phys. Rev. Applied 10, 064026 (2018).

[19] S. Dai, L. Liu, D. Han, and J. Zi, Phys. Rev. B 98, 081405(R) (2018).

[20] Y. He, G. Guo, T. Feng, Y. Xu, and A. E. Miroshnichenko, Phys. Rev. B 98, 161112(R) (2018).

[21] J. Jin, X. Yin, L. Ni, M. Soljačić, B. Zhen, and C. Peng, arXiv:1812.00892.

[22] K. Koshelev, S. Lepeshov, M. Liu, A. Bogdanov, and Y. Kivshar, Phys. Rev. Lett. 121, 193903 (2018).

[23] K. Koshelev, A. Bogdanov, and Y. Kivshar, Sci. Bull. 64, 836 (2019).

[24] W. Chen, Y. Chen, and W. Liu, Phys. Rev. Lett. 122, 153907 (2019).

[25] A. Cerjan, C. W. Hsu, and M. C. Rechtsman, Phys. Rev. Lett. 123, 023902 (2019).

[26] K. Koshelev, G. Favraud, A. Bogdanov, Y. Kivshar, and A. Fratalocchi, Nanophotonics 8, 725 (2019).

[27] Z. Sadrieva, K. Frizyuk, M. Petrov, Y. Kivshar, and A. Bogdanov, arXiv:1903.00309.

[28] H. Zhou, C. Peng, Y. Yoon, C. W. Hsu, K. A. Nelson, L. Fu, J. D. Joannopoulos, M. Soljačić, and B. Zhen, Science 359, 1009 (2018).

[29] J. F. Nye, Natural Focusing and Fine Structure of Light (CRC Press/Taylor and Francis group, London, 1999).

[30] M. Berry and M. Dennis, Proc. R. Soc. Ser. A 457, 141 (2001).

[31] M. Dennis, Opt. Commun. 213, 201 (2002).

[32] I. Freund, Opt. Commun. 201, 251 (2002).

[33] R. W. Schoonover and T. D. Visser, Opt. Express 14, 5733 (2006). 
[34] M. Burresi, R. J. P. Engelen, A. Opheij, D. van Oosten, D. Mori, T. Baba, and L. Kuipers, Phys. Rev. Lett. 102, 033902 (2009).

[35] D. Song, D. Leykam, J. Su, X. Liu, L. Tang, S. Liu, J. Zhao, N. K. Efremidis, J. Xu, and Z. Chen, Phys. Rev. Lett. 122, 123903 (2019).

[36] K. Y. Bliokh, M. A. Alonso, and M. R. Dennis, arXiv: 1903.01304.

[37] A. DErrico, M. Maffei, B. Piccirillo, C. de Lisio, F. Cardano, and L. Marrucci, Sci. Rep. 7, 40195 (2017).

[38] E. Otte, C. Alpmann, and C. Denz, Laser Photonics Rev. 12, 1700200 (2018).

[39] Z. F. Sadrieva, I. S. Sinev, K. L. Koshelev, A. Samusev, I. V. Iorsh, O. Takayama, R. Malureanu, A. A. Bogdanov, and A. V. Lavrinenko, ACS Photonics 4, 723 (2017).

[40] See Supplemental Material at http://link.aps.org/ supplemental/10.1103/PhysRevLett.123.116104 for notes and figures about (1) Simulation result about the relation between the radiation quality factor and the asymmetric parameter, (2) Maps of polarization states in the full first Brillouin zone, (3) General temporal coupled mode theory analysis of 2D photonic crystal (PHC) slabs with mirror symmetry, (4) Reflectance (\& transmittance) of 2D PHC slabs with mirror symmetry under circularly polarized incidence, (5) Retrieval of polarization states from the experimental spectra, (6) Detailed spectra under RCP incidence, (7) Effect of substrate on C-points and impacts of different types of symmetry breaking on both at- \& off- $\Gamma$ BICs, (8) An example of modulating the ellipticity of light using a 2D PHC slab: generating circularly polarized light from linearly polarized light.

[41] X. Yin, Z. Ye, J. Rho, Y. Wang, and X. Zhang, Science 339, 1405 (2013).

[42] K. Y. Bliokh, F. J. Rodríguez-Fortuño, F. Nori, and A. V. Zayats, Nat. Photonics 9, 796 (2015). 